\title{
Symmetries and Properties of the Energy-Casimir Mapping in the Ball-Plate Problem
}

\author{
Cristian Lăzureanu and Tudor Bînzar \\ Department of Mathematics, Politehnica University of Timişoara, Piaţa Victoriei Nr. 2, 300006 Timişoara, Romania
}

Correspondence should be addressed to Tudor Bînzar; tudor.binzar@upt.ro

Received 3 August 2016; Revised 15 November 2016; Accepted 28 December 2016; Published 18 January 2017

Academic Editor: Juan C. Marrero

Copyright (C) 2017 Cristian Lăzureanu and Tudor Bînzar. This is an open access article distributed under the Creative Commons Attribution License, which permits unrestricted use, distribution, and reproduction in any medium, provided the original work is properly cited.

In this paper a system derived by an optimal control problem for the ball-plate dynamics is considered. Symplectic and Lagrangian realizations are given and some symmetries are studied. The image of the energy-Casimir mapping is described and some connections with the dynamics of the considered system are presented.

\section{Introduction}

In 1983 the problem of optimal rolling of a sphere over a horizontal plane without slipping was posed by Hammersley [1]. Using quaternion calculus of variations and optimal control theory, Arthurs and Walsh [2] obtained a system of differential equations that can be integrated analytically in terms of elliptic integrals.

A similar problem, named the ball-plate problem, was considered by Brockett and Dai [3]. They have considered the problem of optimal rolling of a ball without slipping between two horizontal plates, the lower plate fixed, and the upper plate mobile. More precisely, by moving of the upper plate, the ball is rolling from the prescribed initial state into some terminal state along a path which minimizes $c \int_{0}^{T}\|\mathbf{V}(t)\|^{2} d t$ among all possible path which satisfy these boundary conditions, where $\mathbf{V}(t)$ is the velocity of the moving plate, $T$ is the time of transfer, and $c$ is a positive constant. The control is the velocity of the center of the ball. The state of such a system is described by the position of the center of the ball and by the orientation of the ball in the threedimensional space. The nonslipping assumptions mean that the instantaneous velocity of the point of contact of the ball with the lower plate is equal to zero, and the velocity of the point of the contact with the moving plate is equal to the velocity of the moving plate. Such kind of motion is of great interest in mechanics, robotics, and control theory [4-7], making it a widely investigated problem.

The ball-plate problem has been formulated by Jurdjevic [8] as an optimal control problem on the Lie group $\mathbb{R}^{2} \times$ $\mathrm{SO}(3)$. Among the results obtained by Jurdjevic in his study we state here the equations of motion, their integrals of motion, and the integrability properties. Hamilton-Poisson formulations, a study of stability of the equilibrium points and numerical integration of the system obtained by Jurdjevic were presented in $[9,10]$. We mention other topics regarding the problem of optimal rolling sphere such as exponential map and the corresponding group of symmetries, Maxwell points [11], parametrization of extremal trajectories by using elliptic functions, and asymptotics of extremal trajectories $[12,13]$.

According to the Jurdjevic's paper [8], in the following, the derivation of the equations of motion in the ball-plate problem is recalled.

Let us consider two right-handed orthonormal frames in the space $\mathbb{R}^{3}$. One is a fixed frame $\mathbf{e}_{1}, \mathbf{e}_{2}, \mathbf{e}_{3}$ centered at a point $O$ in the lower plane, with $\mathbf{e}_{3}$ perpendicular to this plane and pointing upward, and the other one is a moving frame $\mathbf{a}_{1}, \mathbf{a}_{2}, \mathbf{a}_{3}$ fixed to the ball at its center. Let $\mathbf{q}=\left(q_{1}, q_{2}, q_{3}\right)$ be the coordinates of a point $A$ on the surface of the ball relative to the fixed frame and let $\mathbf{Q}=\left(Q_{1}, Q_{2}, Q_{3}\right)$ be the coordinates of the same point relative to the moving frame. 
Thus $\mathbf{q}=\mathbf{Q} R$, where $R \in \mathrm{SO}(3)$ denotes the rotation matrix which transforms the coordinates relative to the moving frame onto the coordinates relative to the stationary frame. Also, if the center $C$ of the ball has the coordinates $\mathbf{q}_{0}=$ $\left(x_{1}, x_{2}, 1\right)$ (the radius of the ball is 1$)$ and $\mathbf{r}$ is the coordinates of $\overrightarrow{C A}$, both relative to the fixed frame, then $\mathbf{q}=\mathbf{q}_{0}+\mathbf{r}$. It gives that the motion of the point $A$ is described by a curve $\mathbf{q}(t)=\mathbf{q}_{0}(t)+\mathbf{r}(t)$ relative to the fixed frame, or by a path $\left(\left(x_{1}(t), x_{2}(t)\right), R(t)\right)$ in $\mathbb{R}^{2} \times \mathrm{SO}(3)$, where $\left(x_{1}(t), x_{2}(t)\right)$ describes the motion of the center of the ball relative to $\mathbf{e}_{1}$ and $\mathbf{e}_{2}$, and $\mathbf{r}(t)=\mathbf{Q} R(t)$ for some path $R(t)$ in $\mathrm{SO}(3)$.

Identifying the tangent space of $\mathrm{SO}(3)$ at any point $R$ as the set of all matrices $R \Omega$, where $\Omega$ is an antisymmetric matrix, it follows

$$
\frac{d R}{d t}=R(t)\left(\begin{array}{ccc}
0 & -\omega_{3}(t) & \omega_{2}(t) \\
\omega_{3}(t) & 0 & -\omega_{1}(t) \\
-\omega_{2}(t) & \omega_{1}(t) & 0
\end{array}\right)
$$

for some functions $\omega_{1}(t), \omega_{2}(t), \omega_{3}(t)$. The vector $\omega(t)=$ $\omega_{1}(t) \mathbf{e}_{1}+\omega_{2}(t) \mathbf{e}_{2}+\omega_{3}(t) \mathbf{e}_{3}$ is the angular velocity generated by $R(t)$. Thus

$$
\frac{d \mathbf{q}}{d t}=\left(\frac{d x_{1}}{d t}, \frac{d x_{2}}{d t}, 0\right)+\mathbf{r}(t) \times \omega(t) .
$$

Denoting $\mathbf{V}(t)=\left(2 u_{1}(t), 2 u_{2}(t)\right)$ the velocity of the moving plate and using the nonslipping assumptions, the equations which describe the motion of the ball are

$$
\begin{aligned}
\dot{x}_{1} & =u_{1}, \\
\dot{x}_{2} & =u_{2}, \\
\frac{d R}{d t} & =R\left(\begin{array}{ccc}
0 & -\omega_{3} & -u_{1} \\
\omega_{3} & 0 & -u_{2} \\
u_{1} & u_{2} & 0
\end{array}\right) .
\end{aligned}
$$

Considering the Lie group $\mathbb{R}^{2} \times \mathrm{SO}(3)$ and the corresponding Lie algebra generated by the base $B=\left\{\mathbf{e}_{1}, \mathbf{e}_{2}, A_{1}, A_{2}, A_{3}\right\}$, where

$$
\begin{aligned}
& A_{1}=\left(\begin{array}{ccc}
0 & 0 & 0 \\
0 & 0 & -1 \\
0 & 1 & 0
\end{array}\right), \\
& A_{2}=\left(\begin{array}{ccc}
0 & 0 & 1 \\
0 & 0 & 0 \\
-1 & 0 & 0
\end{array}\right), \\
& A_{3}=\left(\begin{array}{ccc}
0 & -1 & 0 \\
1 & 0 & 0 \\
0 & 0 & 0
\end{array}\right),
\end{aligned}
$$

(3) may be written as a controllable system

$$
\dot{g}=u_{1}\left[\mathbf{E}_{1}(g)-\mathbf{A}_{2}(g)\right]+u_{2}\left[\mathbf{E}_{2}(g)+\mathbf{A}_{1}(g)\right],
$$

where $g=\left(x_{1}(t), x_{2}(t), R(t)\right)$ and $\mathbf{E}_{1}, \mathbf{E}_{2}, \mathbf{A}_{1}, \mathbf{A}_{2}$ are leftinvariant vector fields whose values at the identity are, respectively, equal to $\left(\mathbf{e}_{1}, 0\right),\left(\mathbf{e}_{2}, 0\right),\left(0, A_{1}\right),\left(0, A_{2}\right)$.

The control problem is to transfer the ball from a given initial position and orientation to a prescribed final position and orientation along a path which minimizes the functional $J$

$$
J\left(u_{1}, u_{2}\right)=\frac{1}{2} \int_{0}^{T}\left(u_{1}^{2}+u_{2}^{2}\right) d t
$$

Using the Maximum Principle, Jurdjevic obtained that the controls which minimize $J$ are given by

$$
\begin{aligned}
& u_{1}=k-y, \\
& u_{2}=l+x,
\end{aligned}
$$

where $x$ and $y$ are the solutions of the system

$$
\begin{aligned}
& \dot{x}=z(k-y), \\
& \dot{y}=z(l+x), \\
& \dot{z}=-k x-l y,
\end{aligned}
$$

and $k, l \in \mathbb{R}$.

If $k=l=0$, then $z=z_{0}$ and it is easy to see that the trajectory of the center of the ball is a circle if $z_{0} \neq 0$, respectively, and a line if $z_{0}=0$. In the sequel we assume that $k^{2}+l^{2}>0$.

The paper is organized as follows. At the beginning of the second section we recall Hamilton-Poisson structures of system (8). We give two symplectic realizations of system (8) and their corresponding Lagrangian realizations. In the end of this section we determine some symmetries of the considered system. More precisely, using the Euler-Lagrange's equations that give a Lagrangian realization, we obtain a one-parameter Lie group of transformations that leaves invariant these equations. The third section is dedicated to the energy-Casimir mapping $(\mathscr{E} \mathscr{C})$. We characterize the equilibrium points as critical points of $\mathscr{E} \mathscr{C}$. Moreover, we give a description of the image of the energy-Casimir mapping and we remark that the boundary of this set is the union of the image through $\mathscr{E} \mathscr{C}$ of the stable equilibrium points. Also, we decompose this set into a disjoint union of the connected semialgebraic sets which are related to the image through $\mathscr{E} \mathscr{C}$ of the stable and unstable equilibrium points, obtaining semialgebraic stratifications. From the topological point of view, we prove that the fibers corresponding to the strata of these semialgebraic stratifications are, respectively, stable equilibrium points, homoclinic orbits, and periodic orbits. Moreover, we give parametric representations of these fibers. Also, employing energetic methods based on Lyapunov functions, we prove some known stability results and new ones. In the last section of this paper, using the parametric representations of the aforementioned fibers, we mention parametric equations of the trajectory of the center of the ball. 


\section{Hamiltonian Structures, Lagrangian Realizations, and Symmetries}

In this section, we give symplectic and Lagrangian realizations of system (8). Also, some symmetries of the considered system are pointed out.

We recall that the following constants of motion of system (8)

$$
\begin{aligned}
& H_{1}(x, y, z)=\frac{1}{2}(l+x)^{2}+\frac{1}{2}(k-y)^{2}, \\
& H_{2}(x, y, z)=\frac{1}{2}\left(x^{2}+y^{2}+z^{2}\right),
\end{aligned}
$$

were given in [8].

In [9], two Poisson structures

$$
\begin{aligned}
& \Pi_{1}=\left[\begin{array}{ccc}
0 & -z & y \\
z & 0 & -x \\
-y & x & 0
\end{array}\right], \\
& \Pi_{2}=\left[\begin{array}{ccc}
0 & 0 & k-y \\
0 & 0 & l+x \\
-k+y & -l-x & 0
\end{array}\right]
\end{aligned}
$$

were considered. Moreover, system (8) has the following Hamilton-Poisson realizations $\left(\mathbb{R}^{3}, \Pi_{1}, H_{1}\right)$ and $\left(\mathbb{R}^{3}, \Pi_{2}, H_{2}\right)$, with the corresponding Casimirs $C_{1}=H_{2}$ and $C_{2}=H_{1}$, respectively. Also, system (8) admits a family of HamiltonPoisson realizations $\left(\mathbb{R}^{3}, \Pi_{a, b}, H_{c, d}\right)$, where $\Pi_{a, b}=a \Pi_{1}-b \Pi_{2}$ and $H_{c, d}=c H_{2}+d H_{1}, a, b, c, d \in \mathbb{R}, a d-b c=1$. In this configuration the function $\mathrm{C}_{a, b}, \mathrm{C}_{a, b}=a H_{2}+b H_{1}$, is a Casimir.

Since $\Pi_{1} \nabla H_{1}=\Pi_{2} \nabla H_{2}$, we can conclude that system (8) is a bi-Hamiltonian system.

In the sequel we present two symplectic realizations of system (8).

Theorem 1. The Hamilton-Poisson mechanical system $\left(\mathbb{R}^{3}\right.$, $\left.\Pi_{2}, H_{2}\right)$ has a full symplectic realization $\left(\mathbb{R}^{4}, \omega, \widetilde{H}_{2}\right)$, where $\omega=d p_{1} \wedge d q_{1}+d p_{2} \wedge d q_{2}$ and

$$
\widetilde{H}_{2}=\frac{1}{2} p_{1}^{2}+\frac{1}{2} p_{2}^{2}+\left(k \sin q_{1}-l \cos q_{1}\right) p_{2} .
$$

Proof. Denoting

$$
\begin{aligned}
& x=p_{2} \cos q_{1}-l, \\
& y=p_{2} \sin q_{1}+k, \\
& z=p_{1},
\end{aligned}
$$

the mapping $\varphi: \mathbb{R}^{4} \rightarrow \mathbb{R}^{3}, \varphi\left(q_{1}, q_{2}, p_{1}, p_{2}\right)=(x, y, z)$, is a surjective submersion. We notice that $\widetilde{H}_{2}=H_{2} \circ \varphi-(1 / 2)\left(k^{2}+\right.$ $l^{2}$ ). For the Hamiltonian $\widetilde{H}_{2}$ the corresponding Hamilton's equations are

$$
\begin{aligned}
& \dot{q}_{1}=p_{1}, \\
& \dot{q}_{2}=p_{2}+k \sin q_{1}-l \cos q_{1}, \\
& \dot{p}_{1}=-k p_{2} \cos q_{1}-l p_{2} \sin q_{1}, \\
& \dot{p}_{2}=0 .
\end{aligned}
$$

Taking into account relations (13), we have

$$
\begin{aligned}
\dot{x} & =\dot{p}_{2} \cos q_{1}-p_{2} \sin q_{1} \dot{q}_{1}=-p_{1} p_{2} \sin q_{1} \\
& =z(k-y), \\
\dot{y} & =\dot{p}_{2} \sin q_{1}+p_{2} \cos q_{1} \dot{q}_{1}=z(x+l) \\
\dot{z} & =\dot{p}_{1}=-k x-l y,
\end{aligned}
$$

whence (13) are mapped onto (8). Moreover, we have

$$
\begin{aligned}
\{x, y\}_{\omega}= & \frac{\partial x}{\partial q_{1}} \cdot \frac{\partial y}{\partial p_{1}}-\frac{\partial x}{\partial p_{1}} \cdot \frac{\partial y}{\partial q_{1}}+\frac{\partial x}{\partial q_{2}} \cdot \frac{\partial y}{\partial p_{2}}-\frac{\partial x}{\partial p_{2}} \\
& \frac{\partial y}{\partial q_{2}}=0=\{x, y\}_{\Pi_{2}}, \\
\{x, z\}_{\omega}= & -p_{2} \sin q_{1}=k-y=\{x, z\}_{\Pi_{2}} \\
\{y, z\}_{\omega}= & p_{2} \cos q_{1}=x+l=\{y, z\}_{\Pi_{2}} ;
\end{aligned}
$$

that is, the canonical structure $\{\cdot, \cdot\}_{\omega}$ induced by $\omega$ is mapped onto the Poisson structure $\Pi_{2}$.

Therefore $\left(\mathbb{R}^{4}, \omega, \widetilde{H}_{2}\right)$ is a full symplectic realization of the Hamilton-Poisson mechanical system $\left(\mathbb{R}^{3}, \Pi_{2}, H_{2}\right)$.

We remark that $\left(H_{1} \circ \varphi\right)\left(q_{1}, q_{2}, p_{1}, p_{2}\right)=(1 / 2) p_{2}^{2}$ is a first integral for system (13).

Theorem 2. The Hamilton-Poisson mechanical system $\left(\mathbb{R}^{3}\right.$, $\left.\Pi_{1,-1}, H_{1}\right)$ has a full symplectic realization $\left(\mathbb{R}^{4}, \omega, \widetilde{H}_{1}\right)$, where $\omega=d p_{1} \wedge d q_{1}+d p_{2} \wedge d q_{2}$ and

$$
\begin{array}{r}
\widetilde{H}_{1}=\frac{1}{2}\left(k p_{1}-l\right)^{2}+\frac{1}{2}\left(\frac{1}{2 k} q_{1}^{2}+l p_{1}+\frac{1}{k} p_{2}+k\right)^{2}, \\
k \neq 0 .
\end{array}
$$

Proof. The corresponding Hamilton's equations are

$$
\begin{aligned}
& \dot{q}_{1}=\frac{l}{2 k} q_{1}^{2}+\left(k^{2}+l^{2}\right) p_{1}+\frac{l}{k} p_{2}, \\
& \dot{q}_{2}=\frac{1}{2 k^{2}} q_{1}^{2}+\frac{l}{k} p_{1}+\frac{1}{k^{2}} p_{2}+1, \\
& \dot{p}_{1}=-\frac{1}{2 k^{2}} q_{1}^{3}-\frac{l}{k} q_{1} p_{1}-\frac{1}{k^{2}} q_{1} p_{2}-q_{1}, \\
& \dot{p}_{2}=0 .
\end{aligned}
$$


As in the proof of Theorem 1, one obtains that the application $\phi: \mathbb{R}^{4} \rightarrow \mathbb{R}^{3}$,

$$
\begin{aligned}
\phi\left(q_{1}, q_{2}, p_{1}, p_{2}\right) & =(x, y, z) \\
& =\left(-k p_{1},-\frac{1}{2 k} q_{1}^{2}-l p_{1}-\frac{1}{k} p_{2}, q_{1}\right),
\end{aligned}
$$

is a surjective submersion, (17) are mapped onto (8), and the structure $\{\cdot, \cdot\}_{\omega}$ induced by $\omega$ is mapped onto the Poisson structure $\Pi_{1,-1}$. Also, $H_{1} \circ \phi=\widetilde{H}_{1}$, which finishes the proof.

Remark 3. For the Poisson structure $\Pi_{1,-1}$, the center of the Poisson algebra $\mathscr{C}^{\infty}\left(\mathbb{R}^{3}, \mathbb{R}\right)$ is generated by the Casimir $C_{1,-1}$, $C_{1,-1}(x, y, z)=(1 / 2) z^{2}-l x+k y-(1 / 2)\left(k^{2}+l^{2}\right)$. We notice that $\left(C_{1,-1} \circ \phi\right)\left(q_{1}, q_{2}, p_{1}, p_{2}\right)=-p_{2}-(1 / 2)\left(k^{2}+l^{2}\right)$ is a first integral for system (17).

Remark 4. Considering the first integrals $\widetilde{H}_{2}$ and $H_{1} \circ \varphi$, respectively, $\widetilde{H}_{1}$ and $C_{1,-1} \circ \phi$, it is easy to see that system (13) and system (17) respectively are completely integrable in the sense of Arnold-Liouville.

We mention that, in the particular case $k=1, l=0$, a symplectic realization and its complete integrability were given in [14].

In the next two results, we give Lagrangian realizations of the aforementioned Hamiltonian mechanical systems.

Proposition 5. The Hamiltonian mechanical system $\left(\mathbb{R}^{4}, \omega\right.$, $\widetilde{H}_{2}$ ) considered in Theorem 1 has a Lagrangian realization on the tangent bundle $T \mathbb{R}^{2}$ given by the Euler-Lagrange's equations

$$
\begin{aligned}
& \ddot{q}_{1}=\left(k \sin q_{1}-l \cos q_{1}-\dot{q}_{2}\right)\left(k \cos q_{1}+l \sin q_{1}\right), \\
& \ddot{q}_{2}=\dot{q}_{1}\left(k \cos q_{1}+l \sin q_{1}\right),
\end{aligned}
$$

generated by the Lagrangian

$$
L=\frac{1}{2} \dot{q}_{1}^{2}+\frac{1}{2}\left(\dot{q}_{2}-k \sin q_{1}+l \cos q_{1}\right)^{2} .
$$

Proof. For the Lagrangian $L(20)$ the Euler-Lagrange equations $(d / d t)\left(\partial L / \partial \dot{q}_{i}\right)-\partial L / \partial q_{i}=0, i \in\{1,2\}$ become (19). Also, differentiating equations (13) one obtains (19). It is easy to see that $\widetilde{H}_{2}=p_{1} \dot{q}_{1}+p_{2} \dot{q}_{2}-L$, where $p_{i}=\partial L / \partial \dot{q}_{i}, i \in\{1,2\}$, which finishes the proof.

Analogously one obtains the following.

Proposition 6. The Hamiltonian mechanical system $\left(\mathbb{R}^{4}, \omega\right.$, $\left.\widetilde{H}_{1}\right)$ considered in Theorem 2 has a Lagrangian realization on the tangent bundle $T \mathbb{R}^{2}$ given by the Euler-Lagrange's equations

$$
\begin{aligned}
k \ddot{q}_{1}-l q_{1} \dot{q}_{1}+k\left(k^{2}+l^{2}\right) q_{1} \dot{q}_{2} & =0, \\
k^{2} \ddot{q}_{2}-q_{1} \dot{q}_{1}+k l q_{1} \dot{q}_{2} & =0,
\end{aligned}
$$

generated by the Lagrangian

$$
\begin{aligned}
L= & \frac{1}{2 k^{2}} \dot{q}_{1}^{2}+\frac{k^{2}+l^{2}}{2} \dot{q}_{2}^{2}-\frac{l}{k} \dot{q}_{1} \dot{q}_{2}+\frac{l}{k} \dot{q}_{1} \\
& -\left(\frac{1}{2} q_{1}^{2}+k^{2}+l^{2}\right) \dot{q}_{2} .
\end{aligned}
$$

In the last part of this section we study some symmetries of system (8). For this purpose, we will use the Lagrangian realization given in Proposition 6.

A symmetry group of a system of differential equations is a group of transformations which maps any solution to another solution of the system. Following [15], let us search for a one-parameter $(\epsilon)$ Lie group of transformations

$$
\begin{aligned}
& t^{*}=t+\epsilon \xi\left(t, q_{1}, q_{2}\right)+\mathcal{O}\left(\epsilon^{2}\right), \\
& q_{1}^{*}=q_{1}+\epsilon \eta_{1}\left(t, q_{1}, q_{2}\right)+\mathcal{O}\left(\epsilon^{2}\right), \\
& q_{2}^{*}=q_{2}+\epsilon \eta_{2}\left(t, q_{1}, q_{2}\right)+\mathcal{O}\left(\epsilon^{2}\right),
\end{aligned}
$$

which leaves (21) invariant. The corresponding infinitesimal generator is given by a vector field $\mathbf{v}$ :

$$
\begin{aligned}
\mathbf{v}= & \xi\left(q_{1}, q_{2}, t\right) \frac{\partial}{\partial t}+\eta_{1}\left(q_{1}, q_{2}, t\right) \frac{\partial}{\partial q_{1}} \\
& +\eta_{2}\left(q_{1}, q_{2}, t\right) \frac{\partial}{\partial q_{2}},
\end{aligned}
$$

with the property that the action of its second prolongation on (21) vanishes. The second prolongation of the vector field $\mathbf{v}$ is given by

$$
\begin{aligned}
\operatorname{pr}^{(2)}(\mathbf{v})= & \mathbf{v}+\sum_{i=1}^{2}\left(\dot{\eta}_{i}-\dot{\xi} \dot{q}_{i}\right) \frac{\partial}{\partial \dot{q}_{i}} \\
& +\sum_{i=1}^{2}\left(\ddot{\eta}_{i}-\ddot{\xi} \dot{q}_{i}-2 \dot{\xi} \ddot{q}_{i}\right) \frac{\partial}{\partial \ddot{q}_{i}} .
\end{aligned}
$$

A method to find $\mathbf{v}$ is given, for example, in $[15,16]$. More precisely, taking into account the chain rule for the computation of $\dot{\xi}, \ddot{\xi}, \dot{\eta}_{1}, \ddot{\eta}_{1}, \dot{\eta}_{2}, \ddot{\eta}_{2}$ and replacing $\ddot{q}_{1}, \ddot{q}_{2}$ by using (21), the relations obtained by applying the second prolongation of $\mathbf{v}$ on (21) become two equations in $t, q_{1}, \dot{q}_{1}, q_{2}, \dot{q}_{2}$, which are all independent. These equations must be satisfied identically in $t, q_{1}, \dot{q}_{1}, q_{2}, \dot{q}_{2}$, which leads to the finding of $\mathbf{v}$. Detailed utilization of the aforementioned method can be found, for example, in $[17,18]$.

Proposition 7. The infinitesimal generator of (23) is given by

$$
\mathbf{v}=(\alpha t+\beta) \frac{\partial}{\partial t}-\alpha q_{1} \frac{\partial}{\partial q_{1}}-\left(\alpha q_{2}-\gamma\right) \frac{\partial}{\partial q_{2}},
$$

where $\alpha, \beta, \gamma \in \mathbb{R}$.

Proof. It is easy to see that the action of $\operatorname{pr}^{2}(\mathbf{v})$ on (21) vanishes, where $\mathbf{v}$ is given by (26). 
Now, some variational symmetries of system (21) can be presented.

Remark 8. (i) For $\alpha=\beta=0$ and $\gamma \neq 0$, we have $\mathbf{v}_{1}=\gamma\left(\partial / \partial q_{2}\right)$ that represents a translation in the cyclic $q_{2}$ direction which is related to the conservation of $\left(C_{1,-1} \circ \phi\right)\left(q_{1}, q_{2}, p_{1}, p_{2}\right)=$ $-p_{2}-(1 / 2)\left(k^{2}+l^{2}\right)$ given in Remark 3 .

(ii) For $\alpha=\gamma=0$ and $\beta \neq 0$, we have $\mathbf{v}_{2}=\beta(\partial / \partial t)$ that represents the time translation symmetry which generates the conservation of energy $\widetilde{H}_{1}$ given by (16).

Also, since $\operatorname{pr}^{(1)}\left(\mathbf{v}_{j}\right)(L)+L\left(d \xi_{j} / d t\right)=0, j \in\{1,2\}$, where

$$
\operatorname{pr}^{(1)}(\mathbf{v})=\mathbf{v}+\left(\dot{\eta}_{1}-\dot{\xi}_{\dot{q}_{1}}\right) \frac{\partial}{\partial \dot{q}_{1}}+\left(\dot{\eta}_{2}-\dot{\xi} \dot{q}_{2}\right) \frac{\partial}{\partial \dot{q}_{2}},
$$

and $L$ is given by (22), $\mathbf{v}_{1}$ and $\mathbf{v}_{2}$ are variational symmetries.

Remark 9. Taking into account (26), the generators are $\xi=$ $\alpha t+\beta, \eta_{1}=-\alpha q_{1}$, and $\eta_{2}=-\alpha q_{2}+\gamma$, which means that a threeparameter group of symmetries was obtained. Denoting $\mathbf{e}_{1}=$ $\partial / \partial q_{2}, \mathbf{e}_{2}=\partial / \partial t$, and $\mathbf{e}_{3}=t(\partial / \partial t)-q_{1}\left(\partial / \partial q_{1}\right)-q_{2}\left(\partial / \partial q_{2}\right)$, the 3-dimensional Lie algebra of symmetries of (21) endowed with the standard Lie bracket vector fields is generated by the base $\left\{\mathbf{e}_{1}, \mathbf{e}_{2}, \mathbf{e}_{3}\right\}$. Since $\left[\mathbf{e}_{1}, \mathbf{e}_{2}\right]=0,\left[\mathbf{e}_{2}, \mathbf{e}_{3}\right]=\mathbf{e}_{2},\left[\mathbf{e}_{3}, \mathbf{e}_{1}\right]=\mathbf{e}_{1}$, this Lie algebra is of type $\mathrm{V}$ in Bianchi's classification [19].

\section{Properties of the Energy-Casimir Mapping}

It is a well-known fact that the dynamics of a HamiltonPoisson system is foliated by the symplectic leaves associated to the Poisson structure. In [8], Jurdjevic reduced the dynamics (8) to the equation for a pendulum on the level set $\mathscr{C}=\left\{(x, y, z): H_{1}(x, y, z)=1 / 2\right\}$,

$$
\ddot{\theta}+r \sin \theta=0,
$$

where $r=\sqrt{k^{2}+h^{2}}$ (see also [11, 12]).

In [12], Mashtakov and Sachkov gave a partition of the cylinder $\mathscr{C}$ into subsets corresponding to motions of the pendulum (28) of the same type, and using this partition, they introduced elliptic coordinates rectifying the phase flow of the pendulum on a subset of full measure of the cylinder $\mathscr{C}$. Furthermore, parametrization of the solutions of (28) is obtained.

The qualitative information about the Hamiltonian mechanical system $\left(T^{*} \mathbb{R}^{2}, \omega, H\right), \omega=d p_{1} \wedge d q_{1}+d p_{2} \wedge$ $d q_{2}$, can be given by the so-called energy momentum mapping (see, e.g., [15]). A corresponding of the energy momentum mapping in the case of the Hamilton-Poisson system $\left(\mathbb{R}^{3},\{\cdot, \cdot\}, H\right)$ is the energy-Casimir mapping (see, e.g., [20]). In [20], Tudoran et al. formulated the following open questions. "Is there any connection between the dynamical properties of a given dynamical system and the geometry of the image of the energy-Casimir mapping, and if yes, how can one detect as many dynamical elements as possible (e.g., equilibria, periodic orbits, and homoclinic and heteroclinic connections) and dynamical behavior (e.g., stability, bifurcation phenomena for equilibria, periodic orbits, and homoclinic and heteroclinic connections) by just looking at the image of this mapping?" Particularly, for some dynamical systems, the answers to these questions were given (see, e.g., [21-23]).

The goal of this section is to give an answer to the above questions in the case of system (8). Consequently, in this section we study some connections between the dynamics of the considered system and the corresponding energyCasimir mapping. Our approach regards stability problem, homoclinic and periodic orbits, and their connections with the image of the energy-Casimir mapping and the fibers of the energy-Casimir mapping, respectively.

In the following, we consider system (8) with the Hamilton-Poisson realization $\left(\mathbb{R}^{3}, \Pi_{1}, H\right)$, where $\Pi_{1}$ is given by (10) and the Hamiltonian $H=H_{1}$ and a Casimir $C=$ $\mathrm{H}_{2}$, given by (9). Consequently, the energy-Casimir mapping corresponding to the considered system is defined by $\mathscr{E} \mathscr{C}$ : $\mathbb{R}^{3} \rightarrow \mathbb{R}^{2}$

$$
\begin{aligned}
\mathscr{E} \mathscr{C} & (x, y, z)=(H(x, y, z), C(x, y, z)) \\
= & \left(\frac{1}{2}(x+l)^{2}+\frac{1}{2}(y-k)^{2}, \frac{1}{2} x^{2}+\frac{1}{2} y^{2}+\frac{1}{2} z^{2}\right) .
\end{aligned}
$$

Using the critical points of mapping (29), we present a characterization of the equilibrium points of system (8).

Proposition 10. Let $u_{0}:=\left(x_{0}, y_{0}, z_{0}\right) \in \mathbb{R}^{3}$. The following assertions are equivalent:

(i) $u_{0}$ is an equilibrium point of system (8);

(ii) $u_{0}$ is a critical point of energy-Casimir mapping (29);

(iii) $\nabla H\left(u_{0}\right) \times \nabla C\left(u_{0}\right)=\mathbf{0}$.

Proof. Let us observe that system (8) can be written in the equivalent form $\dot{X}=-\nabla H \times \nabla C, X=(x, y, z)^{t}$. Therefore the equilibria of system (8) are given by the condition $(z(k-$ $y), z(l+x),-k x-l y)^{t}=\nabla H \times \nabla C=\mathbf{0}$.

On the other hand, a point $u_{0}:=\left(x_{0}, y_{0}, z_{0}\right) \in \mathbb{R}^{3}$ is a critical point of $\mathscr{E} \mathscr{C}$ if and only if the derivative of $\mathscr{E} \mathscr{C}$ at $u_{0}$ is not surjective; that is, $D \mathscr{E} \mathscr{C}\left(u_{0}\right)$ has rank less than two. Taking into account that

$$
\begin{aligned}
D \mathscr{E} \mathscr{C}(x, y, z) & =\left[\begin{array}{l}
D H(x, y, z) \\
D C(x, y, z)
\end{array}\right] \\
& =\left[\begin{array}{ccc}
x+l & y-k & 0 \\
x & y & z
\end{array}\right],
\end{aligned}
$$

the conclusion follows.

In order to present the connections between the equilibrium points and the image of the energy-Casimir mapping, we give a description of the image of the energy-Casimir mapping. Also, we present a study of the stability of equilibrium points.

By the image of the energy-Casimir mapping $\mathscr{E} \mathscr{C}$ is understood by the set

$$
\begin{aligned}
\operatorname{Im} & (\mathscr{E} \mathscr{C})=\left\{(h, c) \in \mathbb{R}^{2} \mid(\exists)(x, y, z)\right. \\
& \left.\in \mathbb{R}^{3} \text { such that } \mathscr{E} \mathscr{C}(x, y, z)=(h, c)\right\} .
\end{aligned}
$$

Now, we describe the image of the energy-Casimir mapping. 


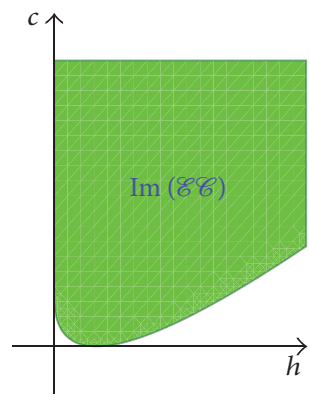

FIgURE 1: The image of the energy-Casimir mapping.

Proposition 11. The image of the energy-Casimir mapping (29) is given by

$$
\begin{gathered}
\operatorname{Im}(\mathscr{E} \mathscr{C})=\left\{(h, c) \in \mathbb{R}^{2} \mid h \geq 0, c \geq h\right. \\
\left.-\sqrt{2 h\left(k^{2}+l^{2}\right)}+\frac{1}{2}\left(k^{2}+l^{2}\right)\right\} .
\end{gathered}
$$

Proof. We are looking for $(h, c) \in \mathbb{R}^{2}$ such that the system

$$
\begin{aligned}
(x+l)^{2}+(y-k)^{2} & =2 h, \\
x^{2}+y^{2}+z^{2} & =2 c
\end{aligned}
$$

has solutions. It is clear $h \geq 0$ and $c \geq 0$. Denoting $x=-l+$ $\sqrt{2 h} \cos \theta, y=k+\sqrt{2 h} \sin \theta$, we obtain

$$
2 c-2 h=z^{2}+k^{2}+l^{2}-2 \sqrt{2 h}(l \cos \theta-k \sin \theta) .
$$

If we choose $\theta_{0}$ such that $\cos \theta_{0}=l / \sqrt{k^{2}+l^{2}}$ and $\sin \theta_{0}=$ $k / \sqrt{k^{2}+l^{2}}$, it gives

$$
\begin{aligned}
z^{2}= & 2 c-2 h+2 \sqrt{2 h\left(k^{2}+l^{2}\right)} \cos \left(\theta+\theta_{0}\right) \\
& -\left(k^{2}+l^{2}\right)
\end{aligned}
$$

which implies the conclusion.

The graphical description of $\operatorname{Im}(\mathscr{E} \mathscr{C})$ is given in Figure 1.

Remark 12. The curve of the equation $c=h-\sqrt{2 h\left(k^{2}+l^{2}\right)}+$ $(1 / 2)\left(k^{2}+l^{2}\right), h \geq 0$, is an arch of the parabola of equation $c^{2}+$ $h^{2}-2 h c-\left(k^{2}+l^{2}\right)(h+c)+(1 / 4)\left(k^{2}+l^{2}\right)^{2}=0$. One gets the image of the energy-Casimir mapping (32) is the disjoint union of the following connected semialgebraic sets (Figure 2):

$$
\begin{aligned}
\Sigma_{1}^{p} & :=\{(h, c) \mid h>0\} \cap\{(h, c) \mid c-h>0\} \\
& \cap\left\{(h, c) \mid c^{2}+h^{2}-2 h c-\left(k^{2}+l^{2}\right)(h+c)\right. \\
& \left.+\frac{1}{4}\left(k^{2}+l^{2}\right)^{2}>0\right\},
\end{aligned}
$$

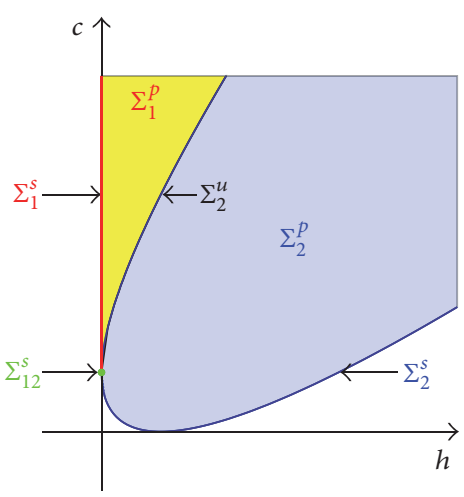

FIGURE 2: The image of the energy-Casimir mapping as a union of connected semialgebraic sets.

$$
\begin{aligned}
\Sigma_{2}^{p} & :=\left\{(h, c) \mid c^{2}+h^{2}-2 h c-\left(k^{2}+l^{2}\right)(h+c)\right. \\
& \left.+\frac{1}{4}\left(k^{2}+l^{2}\right)^{2}<0\right\}, \\
\Sigma_{1}^{s} & :=\{(h, c) \mid h=0\} \cap\left\{(h, c) \mid c-\frac{1}{2}\left(k^{2}+l^{2}\right)>0\right\}, \\
\Sigma_{2}^{s} & :=\left\{(h, c) \mid c^{2}+h^{2}-2 h c-\left(k^{2}+l^{2}\right)(h+c)\right. \\
& \left.+\frac{1}{4}\left(k^{2}+l^{2}\right)^{2}=0\right\} \cap\left\{(h, c) \mid c-h-\frac{1}{2}\left(k^{2}+l^{2}\right)\right. \\
& <0\}, \\
\Sigma_{2}^{u} & :=\left\{(h, c) \mid c^{2}+h^{2}-2 h c-\left(k^{2}+l^{2}\right)(h+c)\right. \\
& \left.+\frac{1}{4}\left(k^{2}+l^{2}\right)^{2}=0\right\} \cap\left\{(h, c) \mid c-h-\frac{1}{2}\left(k^{2}+l^{2}\right)\right. \\
& >0\}, \\
\Sigma_{12}^{s} & :=\{(h, c) \mid h=0\} \cap\left\{(h, c) \mid c-\frac{1}{2}\left(k^{2}+l^{2}\right)=0\right\} .
\end{aligned}
$$

Following, for example, [24], we obtain the following semialgebraic stratifications: $S_{1}:=\Sigma_{1}^{s} \cup \Sigma_{12}^{s} \cup \Sigma_{2}^{u} \cup \Sigma_{1}^{p}$ and $S_{2}:=$ $\Sigma_{2}^{s} \cup \Sigma_{12}^{s} \cup \Sigma_{2}^{u} \cup \Sigma_{2}^{p}$. Here, $\Sigma_{1}^{p}$ and $\Sigma_{2}^{p}$ are principal strata and, as we will see in the following, the superscripts $s$ and $u$ stand, respectively, for stable and unstable.

By Proposition 7 , the union $\mathscr{E}_{1} \cup \mathscr{E}_{2}$ gives the equilibrium points of system (8), where

$$
\begin{aligned}
& \mathscr{E}_{1}=\{(-l, k, M) \mid M \in \mathbb{R}\}, \\
& \mathscr{E}_{2}=\{(-l-l M, k+k M, 0) \mid M \in \mathbb{R}\} .
\end{aligned}
$$

In [10] the stability problem was treated by using the energyCasimir method. However, the stability of the equilibrium points $(0,0,0)$ and $(-l, k, 0)$ was not established. In the 
following we complete the study of the stability. Also, using Lyapunov functions, we give another proof of the known stability results.

Proposition 13 (see, also, [10]). All the equilibrium points from the family $\mathscr{E}_{1}$ are nonlinearly stable.

Proof. Considering the function $L \in \mathscr{C}^{\infty}\left(\mathbb{R}^{3}, \mathbb{R}\right)$,

$$
\begin{aligned}
L(x, y, z)= & (x+l)^{2}+(y-k)^{2} \\
& +\left(x^{2}+y^{2}+z^{2}-k^{2}-l^{2}-M^{2}\right)^{2},
\end{aligned}
$$

we have

$$
\begin{aligned}
& L(-l, k, M)=0, \\
& L(x, y, z)>0, \quad(\forall)(x, y, z) \in V \backslash\{(-l, k, M)\},
\end{aligned}
$$

for some neighborhood $V$ of $(-l, k, M)$. Moreover, using (8), it follows

$$
\begin{aligned}
& \dot{L}(x, y, z)=\frac{\partial L}{\partial x} \cdot \dot{x}+\frac{\partial L}{\partial y} \cdot \dot{y}+\frac{\partial L}{\partial z} \cdot \dot{z}=2(x+l) \dot{x} \\
& +2(y-k) \dot{y}+4\left(x^{2}+y^{2}+z^{2}-k^{2}-l^{2}-M^{2}\right) \\
& \cdot(x \dot{x}+y \dot{y}+z \dot{z})=2 z(x+l)(k-y)+2 z(y-k) \\
& \cdot(x+l)+4\left(x^{2}+y^{2}+z^{2}-k^{2}-l^{2}-M^{2}\right) \\
& \cdot[x z(k-y)+y z(l+x)-(k x+l y) z]=0,
\end{aligned}
$$

for all $(x, y, z) \in V$.

By $[25,26]$, we deduce that all the equilibrium states from the family $\mathscr{E}_{1}$ are nonlinearly stable.

Proposition 14 (see, also, [10]). The equilibrium points from $\mathscr{E}_{2}$ are nonlinearly stable for $M \in(-\infty, 0]$ and unstable otherwise.

Proof. The characteristic polynomial associated with the linear part of system (8) at the equilibrium $e_{M}:=(-l-l M, k+$ $k M, 0) \in \mathscr{E}_{2}$ is given by

$$
p_{e_{M}}(\lambda)=-\lambda\left[\lambda^{2}-M\left(k^{2}+l^{2}\right)\right] .
$$

If $M>0$ we notice that a root of $p_{E_{M}}$ is strictly positive, whence $e_{M}$ is an unstable equilibrium state. Therefore, all the equilibrium states from the family $\mathscr{E}_{2}$ are unstable in the case $M \in(0, \infty)$.

If $M=0$, then $e_{M}=(-l, k, 0) \in \mathscr{E}_{1} \cap \mathscr{E}_{2}$, whence it is nonlinearly stable.

Now, if $M<0$, let us consider the function $L \in$ $\mathscr{C}^{\infty}\left(\mathbb{R}^{3}, \mathbb{R}\right)$,

$$
\begin{aligned}
L(x, y, z)= & {\left[(x+l)^{2}+(y-k)^{2}-M^{2}\left(k^{2}+l^{2}\right)\right]^{2} } \\
& +\left[x^{2}+y^{2}+z^{2}-(M+1)^{2}\left(k^{2}+l^{2}\right)\right]^{2} .
\end{aligned}
$$

It follows $L\left(e_{M}\right)=0, L(x, y, z) \geq 0$ and, using (8), $\dot{L}(x, y, z)=0$ for all $(x, y, z) \in \mathbb{R}^{3}$. Therefore, in order

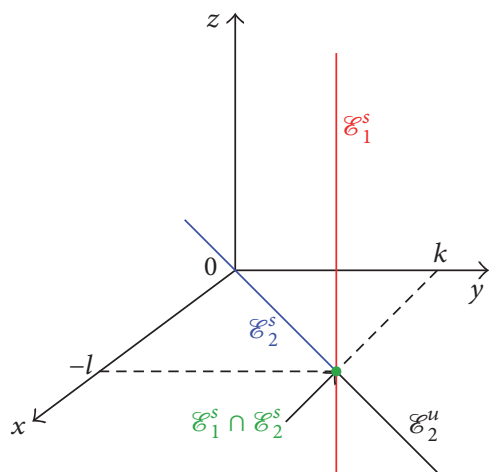

FIgURE 3: The diagram of equilibrium points.

to prove the nonlinear stability of the equilibrium point $e_{M}$, it only remains to prove that the condition $L(x, y, z)=0$ implies $(x, y, z)=e_{M}$; that is, the system

$$
\begin{aligned}
(x+l)^{2}+(y-k)^{2} & =M^{2}\left(k^{2}+l^{2}\right), \\
x^{2}+y^{2}+z^{2} & =(M+1)^{2}\left(k^{2}+l^{2}\right)
\end{aligned}
$$

has a unique solution, $e_{M}$.

If $M=-1$, it is obvious that the above system has a unique solution, namely, $(0,0,0)$. Thus this equilibrium state is nonlinearly stable.

Let now $M \in(-\infty, 0) \backslash\{-1\}$. From geometric point of view, the solution of system (43) is the curve of intersection between a circular cylinder and a sphere. This curve is reducing at a point if and only if the sphere and the cylinder are tangent. In this geometrical configuration, it is enough to prove that the circles obtained by the intersection of the aforementioned surfaces with the plane $x \mathrm{O} y$ are tangent that is the following system has a unique solution:

$$
\begin{aligned}
(x+l)^{2}+(y-k)^{2} & =M^{2}\left(k^{2}+l^{2}\right), \\
x^{2}+y^{2} & =(M+1)^{2}\left(k^{2}+l^{2}\right) .
\end{aligned}
$$

By straightforward computation, one obtains $x=-l-l M$, $y=k+k M$, which finishes the proof.

The diagram of equilibrium points is given in Figure 3.

In the next result we give some connections between the image through the energy-Casimir mapping of the families of equilibrium states and the set $\operatorname{Im}(\mathscr{E} \mathscr{C})$.

Proposition 15. Let $\mathscr{E}_{1}^{s}$ and $\mathscr{E}_{2}^{s}$ and $\mathscr{E}_{2}^{u}$ be the families of nonlinearly stable equilibrium points and unstable equilibrium points, respectively, and let $\mathscr{E} \mathscr{C}$ be the energy-Casimir mapping (29) of system (8). Then,

(i) $\partial \operatorname{Im}(\mathscr{E} \mathscr{C})=\operatorname{Im}\left(\left.\mathscr{E} \mathscr{C}\right|_{\mathscr{E}_{1}^{s}}\right) \cup \operatorname{Im}\left(\left.\mathscr{E} \mathscr{C}\right|_{\mathscr{C}_{2}}\right)$, where $\partial A$ denotes the boundary of the set $A$;

(ii) $\Sigma_{12}^{s}=\operatorname{Im}\left(\left.\mathscr{E} \mathscr{C}\right|_{\mathscr{E}_{1}^{s} \cap \mathscr{E}_{2}^{s}}\right), \Sigma_{1}^{s}=\operatorname{Im}\left(\left.\mathscr{E} \mathscr{C}\right|_{\mathscr{E}_{1}^{s}}\right) \backslash \Sigma_{12}^{s}, \Sigma_{2}^{s}=$ $\operatorname{Im}\left(\left.\mathscr{E} \mathscr{C}\right|_{\mathscr{E}_{2}^{s}}\right) \backslash \Sigma_{12}^{s}$, and $\Sigma_{2}^{u}=\operatorname{Im}\left(\left.\mathscr{E} \mathscr{C}\right|_{\mathscr{C}} u\right)$;

(iii) $\operatorname{Im}(\mathscr{E} \mathscr{C})=\overline{c o}\left\{\operatorname{Im}\left(\left.\mathscr{E} \mathscr{C}\right|_{\mathscr{E}_{1}^{s}}\right), \operatorname{Im}\left(\left.\mathscr{E} \mathscr{C}\right|_{\mathscr{E}_{2}^{s}}\right)\right\}$, where $\overline{c o}$ denotes the convex hull. 


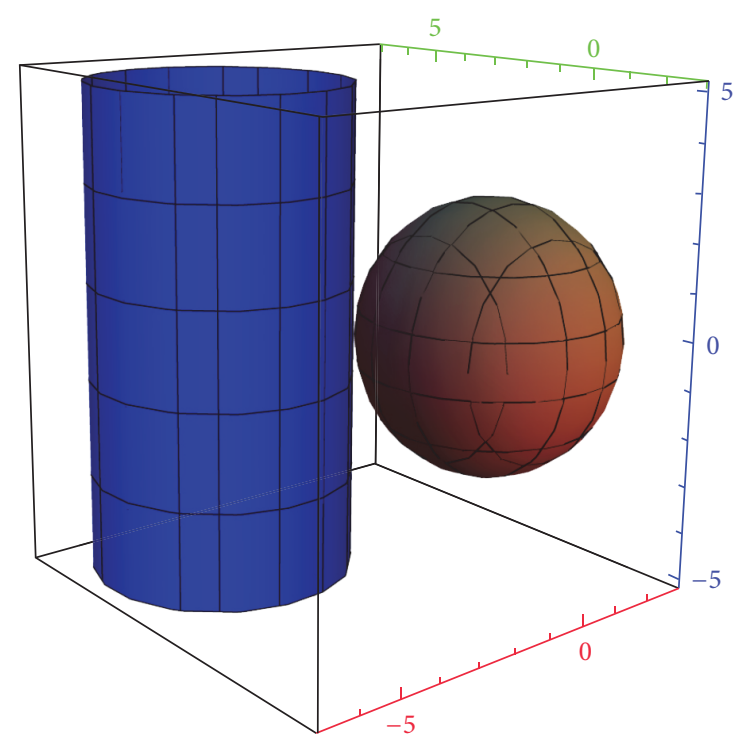

FIgURE 4: The fiber $\mathscr{F}_{(h, c)},(h, c) \in \Sigma_{2}^{s}$ : a stable equilibrium point.

Proof. The conclusions result by simple computations.

Remark 16. We notice that if $e_{M} \in \mathscr{E}_{1}$, then $\operatorname{rank}\left(D \mathscr{E} \mathscr{C}\left(e_{M}\right)\right)=0$ and $\mathscr{E} \mathscr{C}\left(e_{M}\right)$ belongs to a line. Also, if $e_{M} \in \mathscr{E}_{2}$, then $\operatorname{rank}\left(D \mathscr{E} \mathscr{C}\left(e_{M}\right)\right)=1$ and $\mathscr{E} \mathscr{C}\left(e_{M}\right)$ belongs to a curve, namely, a parabola.

In the sequel we study the topology of the fibers of the energy-Casimir mapping (29). A fiber of the energy-Casimir mapping $\mathscr{E} \mathscr{C}$ is the preimage of an element $(h, c) \in \operatorname{Im}(\mathscr{E} \mathscr{C})$ through $\mathscr{E} \mathscr{C}$, that is,

$$
\mathscr{F}_{(h, c)}=\left\{(x, y, z) \in \mathbb{R}^{3} \mid \mathscr{E} \mathscr{C}(x, y, z)=(h, c)\right\} .
$$

By fixing an element $(h, c)$ from $\operatorname{Im}(\mathscr{E} \mathscr{C})$, it belongs to one stratum (36). Therefore the classification of the fibers will be done taking into account the stratification of $\operatorname{Im}(\mathscr{E} \mathscr{C})$. Since the dynamics has place to the intersection between the surfaces $H(x, y, z)=h$ and $C(x, y, z)=c$, one obtains that these fibers are, respectively, a stable equilibrium point, a periodic orbit, a pair of homoclinic orbits, a pair of periodic orbits, and two stable equilibrium points (Figures 4, 5, 6, and 7).

Now we prove the observed results. The first proposition refers to the fibers related to the stable equilibrium states.

Proposition 17. Let $(h, c) \in \operatorname{Im}\left(\left.\mathscr{E} \mathscr{C}\right|_{\mathscr{E}_{1}^{s} \cup \mathscr{C}_{2}^{s}}\right)$. Then the corresponding fiber contains only stable equilibrium points; namely,

(i) if $(h, c) \in \Sigma_{1}^{s}$, then $\mathscr{F}_{(h, c)}=\{(-l, k$, $\left.\left.\pm \sqrt{c-\left(k^{2}+l^{2}\right) / 2}\right)\right\}$

(ii) if $(h, c) \in \Sigma_{2}^{s}$, then $\mathscr{F}_{(h, c)}=\left\{\left(-l+l \sqrt{2 h /\left(k^{2}+l^{2}\right)}, k-\right.\right.$ $\left.\left.k \sqrt{2 h /\left(k^{2}+l^{2}\right)}, 0\right)\right\}$;

(iii) if $(h, c) \in \Sigma_{12}^{s}$, then $\mathscr{F}_{(h, c)}=\{(-l, k, 0)\}$.

Proof. (i) Using (36) and (45), the conclusion immediately follows.

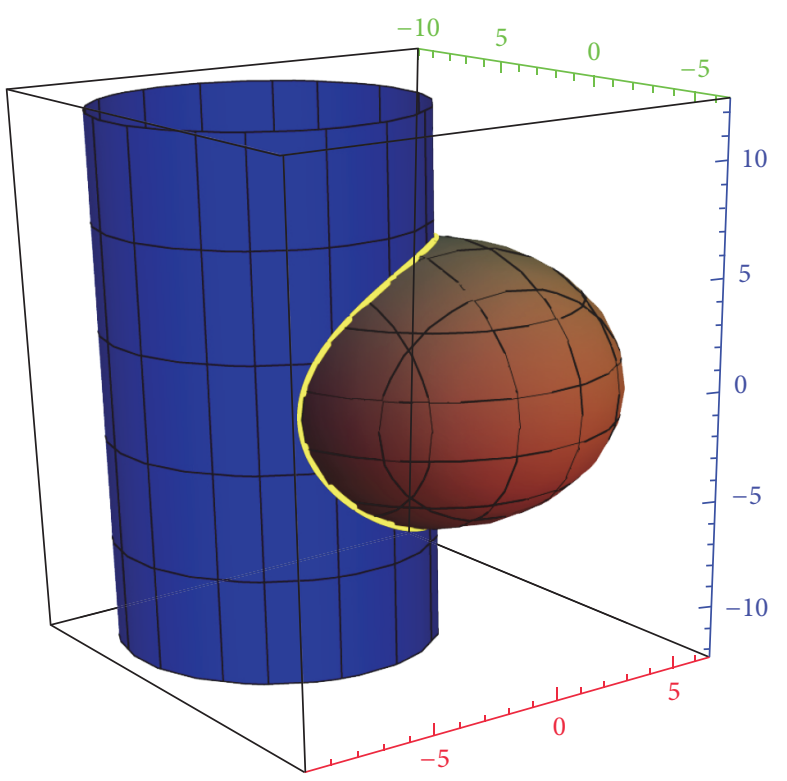

Figure 5: The fiber $\mathscr{F}_{(h, c)},(h, c) \in \Sigma_{2}^{p}$ : a periodic orbit.

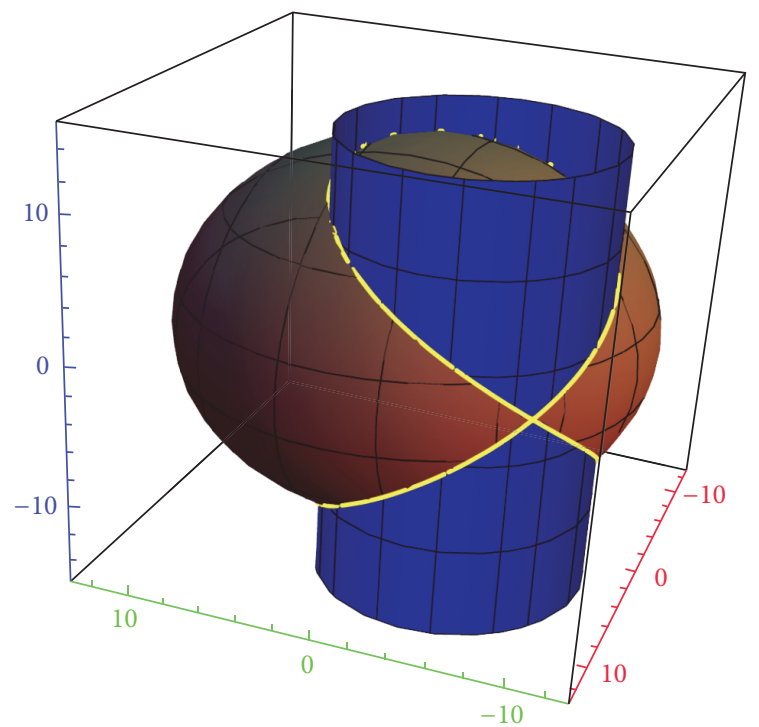

FIgURE 6: The fiber $\mathscr{F}_{(h, c)},(h, c) \in \Sigma_{2}^{u}$ : a pair of homoclinic orbit.

(ii) In this case the fiber is the intersection between the cylinder $(x+l)^{2}+(y-k)^{2}=(\sqrt{2 h})^{2}$ and the sphere $x^{2}+y^{2}+$ $z^{2}=\left(\sqrt{2 h}-\sqrt{k^{2}+l^{2}}\right)^{2}$. Observing that the sphere and the cylinder are tangent (interior or exterior), it follows that $\mathscr{F}_{(h, c)}$ contains only one point, $\left(x_{0}, y_{0}, 0\right)$. Now, by straightforward computation, one obtains the conclusion.

Just looking to the image of the energy-Casimir mapping (Figure 2) and taking into account the papers [20, 22, 23], we expect that there exist homoclinic orbits in dynamics (8). Moreover, Figure 6 suggests that the fibers corresponding to the stratum $\Sigma_{2}^{u}$ are homoclinic orbits. More precisely, we have the following. 
Proposition 18. If $(h, c) \in \Sigma_{2}^{u}$, then $\mathscr{F}_{(h, c)}$ consists of a pair of homoclinic orbits, namely, $\mathscr{F}_{(h, c)}=\left\{\left(x^{+}(t), y^{+}(t), z^{+}(t)\right) \mid t \in\right.$ $\mathbb{R}\} \cup\left\{\left(x^{-}(t), y^{-}(t), z^{-}(t)\right) \mid t \in \mathbb{R}\right\}$, where

$$
\begin{aligned}
& x^{ \pm}(t) \\
& =\sqrt{\frac{2 h}{k^{2}+l^{2}}}\left[ \pm 2 k \frac{\tanh p(t)}{\cosh p(t)}-2 l \tanh ^{2} p(t)+l\right] \\
& \quad-l, \\
& y^{ \pm}(t) \\
& =\sqrt{\frac{2 h}{k^{2}+l^{2}}\left[ \pm 2 l \frac{\tanh p(t)}{\cosh p(t)}+2 k \tanh ^{2} p(t)-k\right]} \\
& \quad+k, \\
& z^{ \pm}(t)= \pm \frac{2 \omega}{\cosh p(t)}
\end{aligned}
$$

with $\omega=\sqrt[4]{2 h\left(k^{2}+l^{2}\right)}, p(t)=\omega\left(t-t_{0}\right), t_{0} \in \mathbb{R}$.

Proof. Following [8], we denote

$$
\begin{aligned}
& x(t)=\sqrt{2 h} \cos \varphi(t)-l, \\
& y(t)=\sqrt{2 h} \sin \varphi(t)+k .
\end{aligned}
$$

It follows $\dot{x}=-\sqrt{2 h} \dot{\varphi} \sin \varphi$. Since $\dot{x}=z(k-y)$, it gives $z=\dot{\varphi}$. Now, taking into account that $C(x, y, z)=c$, one gets

$$
\begin{aligned}
\dot{\varphi}^{2} & =2 c-2 h-\left(k^{2}+l^{2}\right) \\
& +2 \sqrt{2 h\left(k^{2}+l^{2}\right)}\left(\frac{l}{\sqrt{k^{2}+l^{2}}} \cos \varphi\right. \\
& \left.-\frac{k}{\sqrt{k^{2}+l^{2}}} \sin \varphi\right) .
\end{aligned}
$$

If we consider $\varphi_{0}$ such that

$$
\begin{aligned}
& \cos \varphi_{0}=\frac{l}{\sqrt{k^{2}+l^{2}}}, \\
& \sin \varphi_{0}=\frac{k}{\sqrt{k^{2}+l^{2}}}
\end{aligned}
$$

and we denote $\theta=\varphi+\varphi_{0}$, then (48) becomes

$$
\dot{\theta}^{2}=2 c-2 h-\left(k^{2}+l^{2}\right)+2 \sqrt{2 h\left(k^{2}+l^{2}\right)} \cos \theta
$$

and using $(h, c) \in \Sigma_{2}^{u}=\left\{(h, c) \in \mathbb{R}^{2} \mid c=h+\sqrt{2 h\left(k^{2}+l^{2}\right)}+\right.$ $\left.(1 / 2)\left(k^{2}+l^{2}\right)\right\}$, we obtain

$$
\dot{\theta}= \pm 2 \omega \cos \frac{\theta}{2}
$$

where $\omega=\sqrt[4]{2 h\left(k^{2}+l^{2}\right)}$. It follows $\sin (\theta / 2)=\tanh p(t)$, $\cos (\theta / 2)=1 / \cosh p(t)$, with $p(t)=\omega\left(t-t_{0}\right)$, whence



Figure 7: The fiber $\mathscr{F}_{(h, c)},(h, c) \in \Sigma_{1}^{p}$ : a pair of periodic orbit.

$\varphi=2 \arcsin (\tanh p(t))-\varphi_{0}$. Computing $\cos \varphi$ and $\sin \varphi$, we obtain (46).

Now, let us observe that by fixing $(h, c) \in \Sigma_{2}^{u}$ we have $\mathscr{E} \mathscr{C}\left(e_{(h, c)}\right)=(h, c)$, where $e_{(h, c)}=\left(-l+l \sqrt{2 h /\left(k^{2}+l^{2}\right)}, k+\right.$ $\left.k \sqrt{2 h /\left(k^{2}+l^{2}\right)}, 0\right) \in \mathscr{E}_{2}^{u}$. It is easy to see that $\lim _{t \rightarrow-\infty}\left(x^{ \pm}(t)\right.$, $\left.y^{ \pm}(t), z^{ \pm}(t)\right)=\lim _{t \rightarrow \infty}\left(x^{ \pm}(t), y^{ \pm}(t), z^{ \pm}(t)\right)=e_{(h, c)}$, whence the curves given parametrically by (46) are homoclinic orbits.

In the papers $[20-23,27]$ it was reported that the fibers corresponding to the principal strata of the image of the energy-Casimir mapping associated to some particular systems of differential equations are periodic orbits. In our case, Figures 5 and 7 suggest that the fibers corresponding to the principal strata are also periodic orbits. In the following two results we prove the aforementioned connections.

Proposition 19. The fiber $\mathscr{F}_{(h, c)}=\{(x(t), y(t), z(t)) \mid t \in \mathbb{R}\}$ corresponding to a point $(h, c)$ belonging to the principal stratum $\Sigma_{2}^{p}$, where

$$
\begin{aligned}
& x(t)=\sqrt{\frac{2 h}{k^{2}+l^{2}}}\left[2 l \cdot \mathrm{dn}^{2} p(t)+2 m k \cdot \operatorname{sn} p(t)\right. \\
& \cdot \operatorname{dn} p(t)-l]-l, \\
& y(t)=\sqrt{\frac{2 h}{k^{2}+l^{2}}\left[-2 k \cdot \mathrm{dn}^{2} p(t)+2 m l \cdot \operatorname{sn} p(t)\right.} \\
& \cdot \operatorname{dn} p(t)+k]+k, \\
& z(t)=2 m \omega \cdot \operatorname{cn} p(t)
\end{aligned}
$$

with $\omega=\sqrt[4]{2 h\left(k^{2}+l^{2}\right)}, p(t)=\omega\left(t-t_{0}\right), t_{0} \in \mathbb{R}$, and the modulus of the Jacobi's elliptic functions $m=$ $(1 / 2 \omega) \sqrt{2 c-\left(\sqrt{2 h}-\sqrt{k^{2}+l^{2}}\right)^{2}}$, is a periodic orbit. 
Proof. Since $(h, c) \in \Sigma_{2}^{p}$, it follows

$$
-1<\frac{2 h-2 c+k^{2}+l^{2}}{2 \sqrt{2 h\left(k^{2}+l^{2}\right)}}<1 .
$$

Thus there exists $\alpha$ such that

$$
\cos \alpha=\frac{2 h-2 c+k^{2}+l^{2}}{2 \sqrt{2 h\left(k^{2}+l^{2}\right)}} .
$$

Following the steps (47)-(50) from the proof of Proposition 18, (50) becomes

$$
\dot{\theta}^{2}=2 \omega^{2}(\cos \theta-\cos \alpha) .
$$

Therefore the equation of a mathematical pendulum is obtained. Using Jacobi's elliptic functions (see, e.g., [28]) it follows $\cos (\theta / 2)=\operatorname{dn} p(t)$, where $p(t)=\omega\left(t-t_{0}\right)$ and the modulus $m=\sin (\alpha / 2)$. Then $\varphi=2 \arccos (\operatorname{dn} p(t))-\varphi_{0}$. Using the properties of the Jacobi's elliptic functions one gets $z=\dot{\varphi}=2 m \omega \cdot \operatorname{cn} p(t)$. Also, by (47), one obtains $x$ and $y$, which finishes the proof.

Proposition 20. Let $(h, c) \in \Sigma_{1}^{p}$. Then $\mathscr{F}_{(h, c)}=\left\{\left(x^{+}(t), y^{+}(t)\right.\right.$, $\left.\left.z^{+}(t)\right) \mid t \in \mathbb{R}\right\} \cup\left\{\left(x^{-}(t), y^{-}(t), z^{-}(t)\right) \mid t \in \mathbb{R}\right\}$ represents $a$ pair of periodic orbits, where

$$
\begin{aligned}
& x^{ \pm}(t)=\sqrt{\frac{2 h}{k^{2}+l^{2}}}\left[l-2 l \cdot \operatorname{sn}^{2} p(t) \pm 2 k \cdot \operatorname{sn} p(t)\right. \\
& \cdot \operatorname{cn} p(t)]-l, \\
& y^{ \pm}(t)=\sqrt{\frac{2 h}{k^{2}+l^{2}}}\left[-k+2 k \cdot \operatorname{sn}^{2} p(t) \pm 2 l \cdot \operatorname{sn} p(t)\right. \\
& \cdot \operatorname{cn} p(t)]+k, \\
& z^{ \pm}(t)= \pm \frac{2 \omega}{m} \cdot \operatorname{dn} p(t)
\end{aligned}
$$

with $\omega=\sqrt[4]{2 h\left(k^{2}+l^{2}\right)}, p(t)=(\omega / m)\left(t-t_{0}\right), t_{0} \in \mathbb{R}$, and the modulus of the Jacobi's elliptic functions $m=2 \omega /$ $\sqrt{2 c-\left(\sqrt{2 h}-\sqrt{k^{2}+l^{2}}\right)^{2}}$.

Proof. Performing the steps (47)-(50), (50) becomes

$$
\dot{\theta}^{2}=2 \omega^{2}\left(\frac{2}{m^{2}}-1+\cos \theta\right)
$$

where $m=2 \omega / \sqrt{2 c-\left(\sqrt{2 h}-\sqrt{k^{2}+l^{2}}\right)^{2}}$. Because $(h, c) \in \Sigma_{1}^{p}$ it gives $m<1$. We again obtain the equation of a mathematical pendulum and following [28], it follows $\sin (\theta / 2)=\operatorname{sn} p(t)$, where $p(t)=(\omega / m)\left(t-t_{0}\right)$. By straightforward computation the parametrization (56) is obtained.

\section{The Trajectory of the Center of the Ball}

Using a connection with the elastica problem, Jurdjevic [8] gave a completely geometric analysis of the motion of the center of the ball; he described various qualitative types of rolling for a sphere along elastics of various forms: inflectional, noninflectional, a circle, and a straight line.

In [12], using the coordinates obtained by integration of the pendulum motion on the partition of a cylinder, a parametrization of extremal trajectories is obtained.

In this section, we classify the types of the trajectory of the center of the ball taking into account the fibers of the energyCasimir mapping. Using the parametric representations of the studied fibers, we obtain the parametric representations of the trajectory of the center of the ball. These representations are similarly with the ones discussed in [8], respectively [12].

As we have seen in the Introduction, namely, (3) and (7), the controlled motion of the center of the ball is described by the following system:

$$
\begin{aligned}
\dot{x}_{1} & =k-y, \\
\dot{x}_{2} & =l+x, \\
x_{1}(0) & =x_{1}^{0}, \\
x_{2}(0) & =x_{2}^{0},
\end{aligned}
$$

where $x$ and $y$ are given by system (8) and $x_{1}^{0}, x_{2}^{0} \in \mathbb{R}$.

In the following, considering that $(x, y, z)$ belongs to a specific fiber $\mathscr{F}_{(h, c)}$, we will integrate system (58):

$$
\begin{aligned}
& x_{1}(t)=\int_{0}^{t}[k-y(s)] d s+x_{1}^{0}, \\
& x_{2}(t)=\int_{0}^{t}[l+x(s)] d s+x_{2}^{0} .
\end{aligned}
$$

Proposition 21. Let $(h, c) \in \Sigma_{1}^{s} \cup \Sigma_{12}^{s}$ and $(x, y, z) \in \mathscr{F}_{(h, c)}$. Then the ball does not move.

Proof. By Proposition 17, it is obvious.

Proposition 22. Let $(h, c) \in \Sigma_{2}^{s}$ and $(x, y, z) \in \mathscr{F}_{(h, c)}$. Then the ball rolls along the line

$$
\begin{aligned}
& x_{1}=k \sqrt{\frac{2 h}{k^{2}+l^{2}}} t+x_{1}^{0}, \\
& x_{2}=l \sqrt{\frac{2 h}{k^{2}+l^{2}}} t+x_{2}^{0} .
\end{aligned}
$$

Proof. By Proposition 17 (ii), it immediately follows.

Proposition 23. If $(x, y, z) \in \mathscr{F}_{(h, c)}$, where $(h, c) \in \Sigma_{2}^{u}$, then the trajectory of the center of the ball is given by $\left\{\left(x_{1}^{+}(t), x_{2}^{+}(t)\right)\right.$, $t \geq 0\}$ or $\left\{\left(x_{1}^{-}(t), x_{2}^{-}(t)\right), t \geq 0\right\}$, where

$$
\begin{aligned}
x_{1}^{ \pm}(t)= & \frac{\omega}{k^{2}+l^{2}}\left[ \pm \frac{2 l}{\cosh \omega t}+2 k \tanh \omega t-k \omega t\right] \\
& \mp \frac{2 l \omega}{k^{2}+l^{2}}+x_{1}^{0},
\end{aligned}
$$




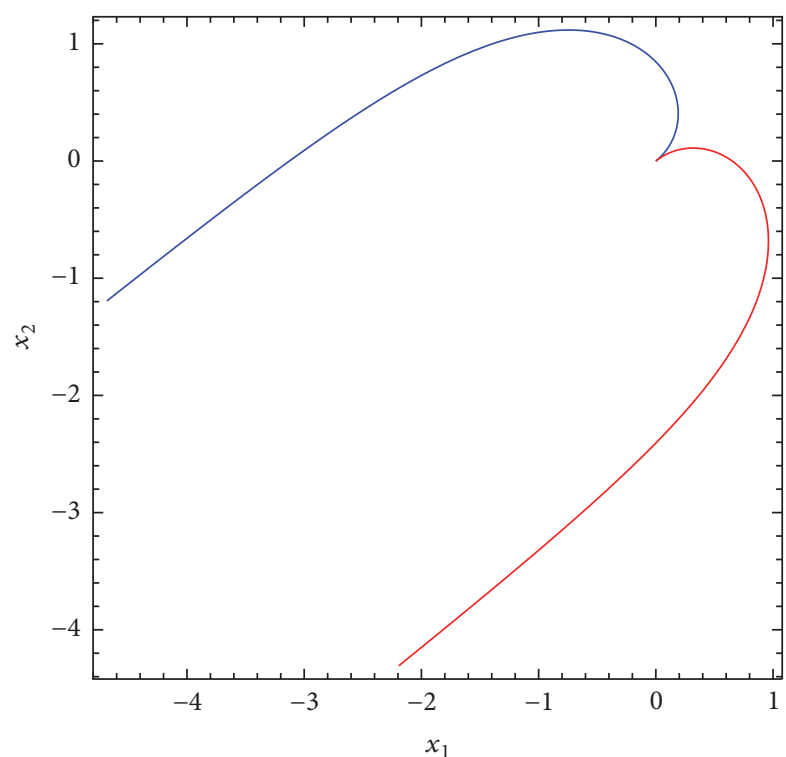

Figure 8: The trajectory of the center of the ball $\left((h, c) \in \Sigma_{2}^{u}, k=5\right.$, $l=4$ ).

$$
\begin{aligned}
x_{2}^{ \pm}(t)= & \frac{\omega}{k^{2}+l^{2}}\left[\mp \frac{2 k}{\cosh \omega t}+2 l \tanh \omega t-l \omega t\right] \\
& \pm \frac{2 k \omega}{k^{2}+l^{2}}+x_{2}^{0}
\end{aligned}
$$

with $\omega=\sqrt[4]{2 h\left(k^{2}+l^{2}\right)}$

Proof. Using (46) with $t_{0}=0$, it is easy to integrate system (58).

Figure 8 presents the situation given in Proposition 23. The blue curve represents the trajectory $\left(x_{1}^{+}(t), x_{2}^{+}(t)\right), t \geq 0$, and the red one the trajectory $\left(x_{1}^{-}(t), x_{2}^{-}(t)\right)$, where $x_{1}^{0}=x_{2}^{0}=$ 0 .

In the following results we are using the Jacobi's epsilon function [28]:

$$
\mathscr{E}(u, m)=\int_{0}^{u} \mathrm{dn}^{2} v d v
$$

where $m$ is the modulus.

Proposition 24. Let $(h, c) \in \sum_{2}^{p}$ and $(x, y, z) \in \mathscr{F}_{(h, c)}$. Then the orbit of the center of the ball is $\left\{\left(x_{1}(t), x_{2}(t)\right), t \geq 0\right\}$, where

$$
\begin{aligned}
x_{1}( & \\
= & \frac{\omega}{k^{2}+l^{2}}[2 k \cdot \mathscr{E}(\omega t, m)+2 l m \cdot \mathrm{cn}(\omega t)-k \omega t] \\
& \quad-\frac{2 l m \omega}{k^{2}+l^{2}}+x_{1}^{0}, \\
x_{2}(t) & \\
= & \frac{\omega}{k^{2}+l^{2}}[2 l \cdot \mathscr{E}(\omega t, m)-2 k m \cdot \operatorname{cn}(\omega t)-l \omega t] \\
& +\frac{2 k m \omega}{k^{2}+l^{2}}+x_{2}^{0}
\end{aligned}
$$

with $\omega=\sqrt[4]{2 h\left(k^{2}+l^{2}\right)}$ and the modulus of the Jacobi's elliptic functions $m=(1 / 2 \omega) \sqrt{2 c-\left(\sqrt{2 h}-\sqrt{k^{2}+l^{2}}\right)^{2}}$.

Proof. Replacing (52) with $t_{0}=0$ in (59) and using (62) and the property $(\operatorname{cn} v)^{\prime}=-\operatorname{sn} v \operatorname{dn} v$, the conclusion follows.

In Figure 9 we present some trajectories of the center of the ball when $h$ is fixed and $c$ increases such that $(h, c) \in \Sigma_{2}^{p}$, first near the stratum $\Sigma_{2}^{s}$ and last near the stratum $\Sigma_{2}^{u}$.

Proposition 25. If $(x, y, z) \in \mathscr{F}_{(h, c)}$, where $(h, c) \in \Sigma_{1}^{p}$, then the orbit of the center of the ball is given by $\left\{\left(x_{1}^{+}(t), x_{2}^{+}(t)\right), t \geq\right.$ $0\}$ (Figure 10, blue) or $\left\{\left(x_{1}^{-}(t), x_{2}^{-}(t)\right), t \geq 0\right\}$ (Figure 10, red), where

$$
\begin{gathered}
x_{1}^{ \pm}(t)=\frac{\omega}{m\left(k^{2}+l^{2}\right)}\left[2 k \cdot \mathscr{E}\left(\frac{\omega t}{m}, m\right) \pm 2 l \cdot \operatorname{dn} \frac{\omega t}{m}\right. \\
\left.-\frac{2 k \omega}{m} t+k m \omega t \mp 2 l\right]+x_{1}^{0}, \\
x_{2}^{ \pm}(t)=\frac{\omega}{m\left(k^{2}+l^{2}\right)}\left[2 l \cdot \mathscr{E}\left(\frac{\omega t}{m}, m\right) \mp 2 k \cdot \operatorname{dn} \frac{\omega t}{m}\right. \\
\left.-\frac{2 l \omega}{m} t+\operatorname{lm} \omega t \pm 2 k\right]+x_{2}^{0}
\end{gathered}
$$

with $\omega=\sqrt[4]{2 h\left(k^{2}+l^{2}\right)}$ and the modulus of the Jacobi's elliptic functions $m=2 \omega / \sqrt{2 c-\left(\sqrt{2 h}-\sqrt{k^{2}+l^{2}}\right)^{2}}$.

Proof. Replacing (56) with $t_{0}=0$ in (59) and taking into account $\operatorname{dn}^{2} v+m^{2} \operatorname{sn}^{2} v=1$, making use of (62) and the property $(\operatorname{dn} v)^{\prime}=-m^{2} \operatorname{sn} v \operatorname{cn} v$, we obtain (64).

In Figure 10 some trajectories of the center of the ball are presented when $h$ is fixed and $c$ increases such that $(h, c) \in \Sigma_{1}^{p}$, first near the stratum $\Sigma_{2}^{u}$.

Remark 26. We notice that the relations (63) and (64) can be written by using elliptic integrals. Indeed, we have $\mathscr{E}(u, m)=$ $E(\operatorname{am} u, m)$ [28], where am $u=\phi$ is the Jacobi amplitude and $E(\phi, m)=\int_{0}^{\phi} \sqrt{1-m^{2} \sin ^{2} \theta} d \theta$ is the elliptic integral of the second kind with the modulus $m$.

Using Wolfram Mathematica software, one can draw a variety of possible trajectories of the center of the ball by customizing $k$ and $l$ and taking $(h, c)$ belonging to different strata.

\section{Conclusions}

In our work some properties of the dynamic of the ballplate problem are studied. We particularly refer here to the connections between the dynamical properties of the considered system of differential equations and the geometrical properties of the corresponding energy-Casimir mapping $\mathscr{E} \mathscr{C}$. Thus, the equilibrium points are the critical points of $\mathscr{E} \mathscr{C}$, and the boundary of the image of the energyCasimir mapping is the image through $\mathscr{E} \mathscr{C}$ of the stable equilibrium states. Also, using the image through $\mathscr{E} \mathscr{C}$ of 

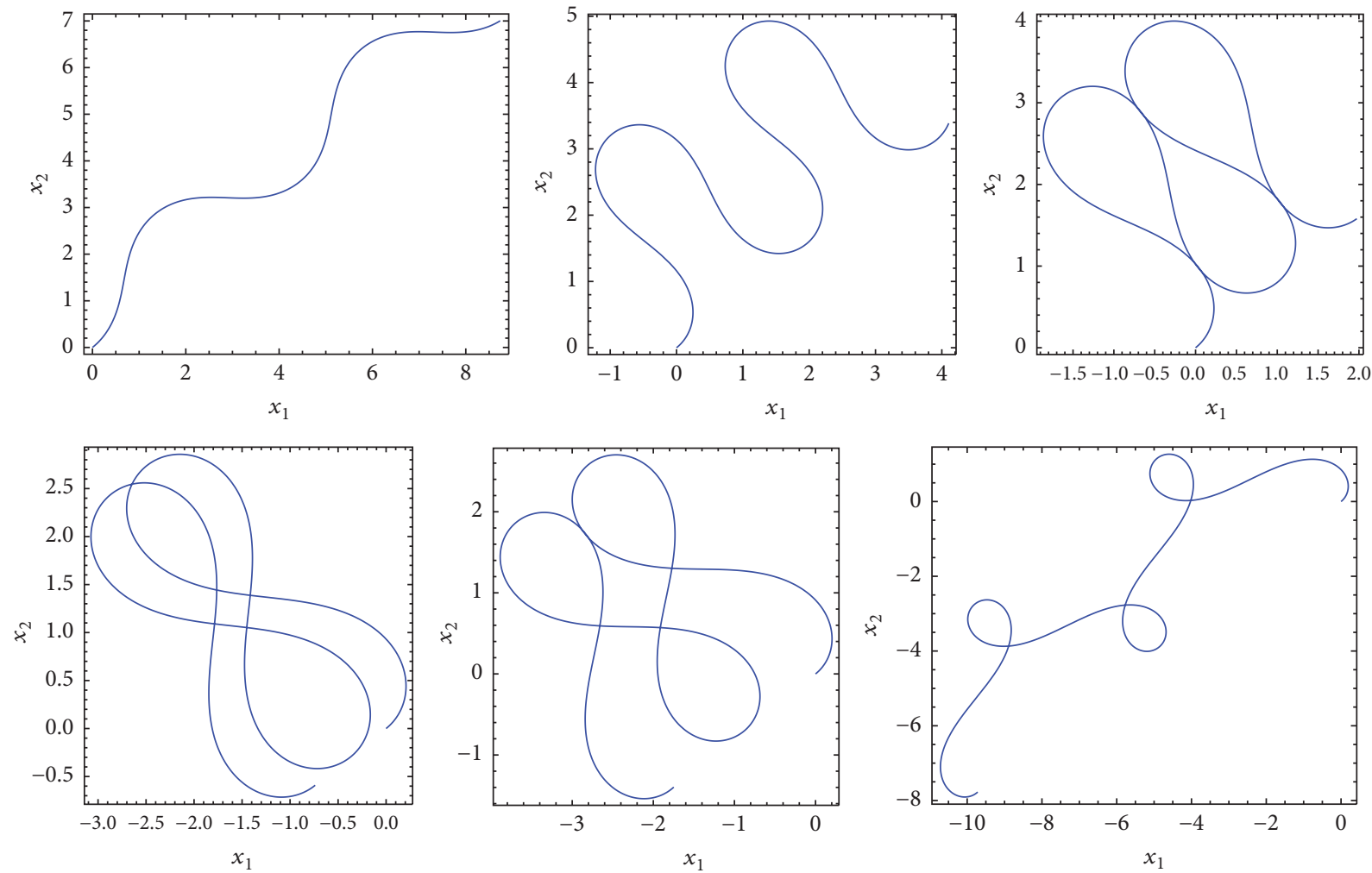

FigURE 9: The trajectory of the center of the ball $\left((h, c) \in \Sigma_{2}^{p}, k=5, l=4, x_{1}^{0}=x_{2}^{0}=0\right)$.
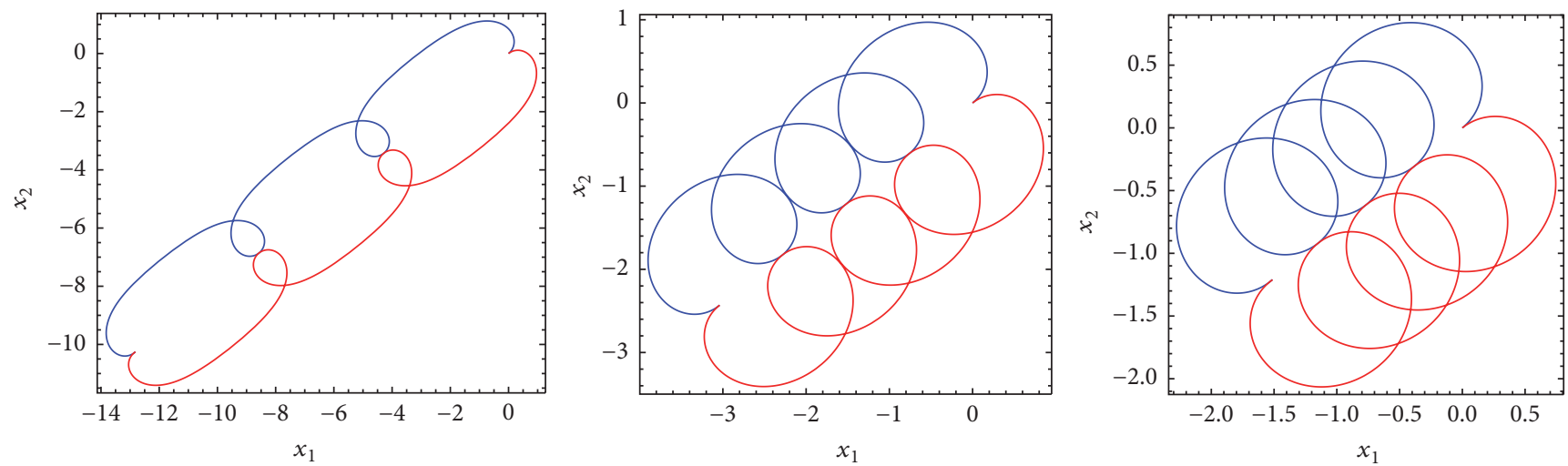

FIGURE 10: The trajectory of the center of the ball $\left((h, c) \in \Sigma_{1}^{p}, k=5, l=4, x_{1}^{0}=x_{2}^{0}=0\right)$.

the equilibrium states, semialgebraic partitions of the image of the energy-Casimir mapping are obtained. In addition, the fibers corresponding to the strata of the presented semialgebraic stratifications are, respectively, stable equilibrium points, homoclinic orbits, and periodic orbits. More precisely, the fibers corresponding to the unstable stratum $\Sigma_{2}^{u}$ are a pair of homoclinic orbits and the fibers corresponding to the principal strata are periodic orbits. It is an open problem to establish a class of three-dimensional system of differential equations with the same properties. In the end of the paper, using the parametric representations of the aforementioned fibers, parametric equations of the trajectory of the center of the ball are mentioned.

\section{Competing Interests}

The authors declare that there is no conflict of interests regarding the publication of this paper.

\section{References}

[1] J. M. Hammersley, "Oxford commemoration ball, Probability, Statistics and Analysis, London Math. Soc. lecture notes," ser, vol. 7, p. 9, 1983.

[2] A. M. Arthurs and G. R. Walsh, "On Hammersley's minimum problem for a rolling sphere," Mathematical Proceedings of the Cambridge Philosophical Society, vol. 99, no. 3, pp. 529-534, 1986. 
[3] R. W. Brockett and L. Dai, "Non-holonomic kinematics and the role of elliptic functions in constructive controllability", in Nonholonomic Motion Planning, Z. Li and J. F. Canny, Eds., pp. 1-23, Kluwer, 1993.

[4] Z. Li and J. Canny, "Motion of two rigid bodies with rolling constraint," IEEE Transactions on Robotics and Automation, vol. 6, no. 1, pp. 62-72, 1990.

[5] A. Bicchi, D. Prattichizzo, and S. S. Sastry, "Planning motions of rolling surfaces," in Proceedings of the 34th IEEE Conference on Decision and Control, pp. 2812-2817, New Orleans, La, USA, December 1995.

[6] A. Marigo and A. Bicchi, "Rolling bodies with regular surface: the holonomic case, differential geometry and control (Boulder, CO 1997)," in Proceedings of Symposia in Pure Mathematics, vol. 64 of American Mathematical Society, pp. 241-256, Providence, RI, USA, 1999.

[7] A. A. Agrachev and Y. L. Sachkov, Control Theory from the Geometric Viewpoint, vol. 87 of Encyclopaedia of Mathematical Sciences, Springer, Berlin, Germany, 2004.

[8] V. Jurdjevic, "The geometry of the plate-ball problem," Archive for Rational Mechanics and Analysis, vol. 124, no. 4, pp. 305-328, 1993.

[9] M. Puta, P. Birtea, C. Lazureanu, C. Pop, and R. Tudoran, Control, Integrability and Stability in Some Concrete Mechanical Problems on Matrix Lie Groups, Dipartamento di Matematica, Universita degli Studi di Roma "La Sapienza”, Roma, Italy, 1998.

[10] C. Pop, A. Aron, and C. Petri, "Geometrical aspects of the ballplate problem," Balkan Journal of Geometry and Its Applications, vol. 16, no. 2, pp. 114-121, 2011.

[11] Y. L. Sachkov, "Maxwell strata and symmetries in the problem of optimal rolling of a sphere over a plane," Sbornik: Mathematics, vol. 201, no. 7, article 1029, 2010.

[12] A. P. Mashatkov and Y. L. Sachkov, "Extremal trajectories and the asymptotics of the Maxwell time in the problem of the optimal rolling of a sphere on a plane," Sbornik: Mathematics, vol. 202, no. 9, pp. 1-25, 2011.

[13] A. P. Mashtakov and A. Y. Popov, "Asymptotics of Maxwell time in the plate-ball problem," Journal of Mathematical Sciences, vol. 195, no. 3, pp. 336-368, 2013.

[14] M. Puta, C. Lăzureanu, and I. Moş, "The complete integrability and geometric prequantization for two optimal control problem," Analele Universităţii de Vest din Timişoara, Seria Matematica-Informatica, vol. 39, no. 2, pp. 133-145, 2001.

[15] G. W. Bluman and S. Kumei, Symmetries and Differential Equations, vol. 81 of Applied Mathematical Sciences, Springer, New York, NY, USA, 1989.

[16] P. J. Olver, Applications of Lie Groups to Differential Equations, vol. 107 of Graduate Texts in Mathematics, Springer, New York, NY, USA, 1986.

[17] C. Lăzureanu and T. Bînzar, "Symmetries of some classes of dynamical systems," Journal of Nonlinear Mathematical Physics, vol. 22, no. 2, pp. 265-274, 2015.

[18] C. Lăzureanu and T. Bînzar, "On some properties and symmetries of the 5-dimensional lorenz system," Mathematical Problems in Engineering, vol. 2015, Article ID 438694, 7 pages, 2015.

[19] L. Bianchi, "Sugli spazi a tre dimensioni che ammettono un gruppo continuo di movimenti," Memorie di Matematica e di Fisica della Societa Italiana delle Scienze, Serie Terza, vol. 11, no. 1898, pp. 267-352, 1898.
[20] R. M. Tudoran, A. Aron, and Ş. Nicoară, "On a Hamiltonian version of the Rikitake system," SIAM Journal on Applied Dynamical Systems, vol. 8, no. 1, pp. 454-479, 2009.

[21] T. Bînzar and C. Lăzureanu, "On some dynamical and geometrical properties of the Maxwell-Bloch equations with a quadratic control," Journal of Geometry and Physics, vol. 70, pp. 1-8, 2013.

[22] T. Bînzar and C. Lăzureanu, "A Rikitake type system with one control," Discrete and Continuous Dynamical Systems, Series B, vol. 18, no. 7, pp. 1755-1776, 2013.

[23] C. Lăzureanu and T. Bînzar, "A Rikitake type system with quadratic control," International Journal of Bifurcation and Chaos, vol. 22, no. 11, Article ID 1250274, 14 pages, 2012.

[24] E. Bierstone and P. Milman, "Semialgebraic and subanalytic sets," Publications Mathématiques de l'IHÉS, vol. 67, pp. 5-42, 1988.

[25] A. M. Lyapunov, "Problème générale de la stabilité du mouvement," Annales de la Faculté des Sciences de Toulouse, vol. 9, pp. 203-474, 1907, Kharkov 1892.

[26] A. M. Lyapunov, Problème Générale de la Stabilité du Mouvement, vol. 17, Princeton University Press, Princeton, NJ, USA, 1949.

[27] C. Lăzureanu and T. Bînzar, "Some geometrical properties of the Maxwell-Bloch equations with a linear control," in Proceedings of the 13th International Conference of Mathematics and Its Applications, pp. 151-158, Timişoara, Romania, November 2012.

[28] D. F. Lawden, Elliptic Functions and Applications, vol. 80 of Applied Mathematical Sciences, Springer, New York, NY, USA, 1989. 


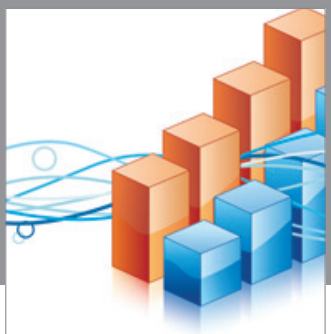

Advances in

Operations Research

vatem alat4



\section{The Scientific} World Journal
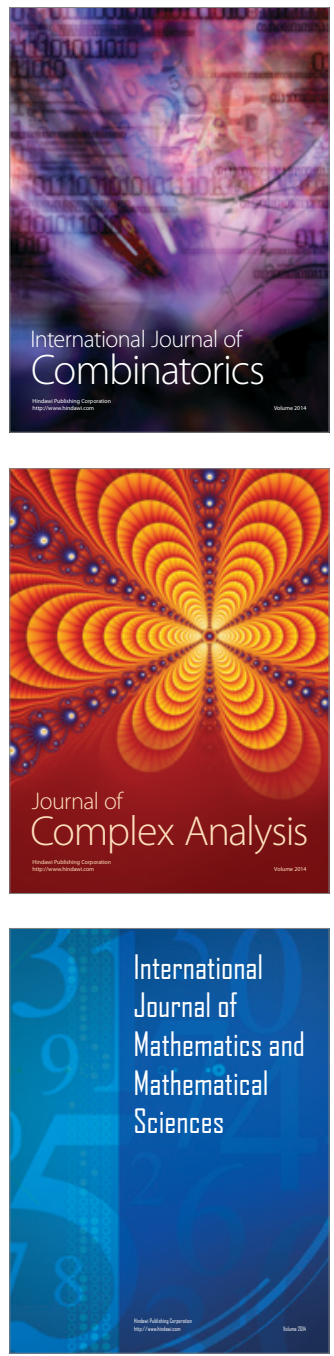
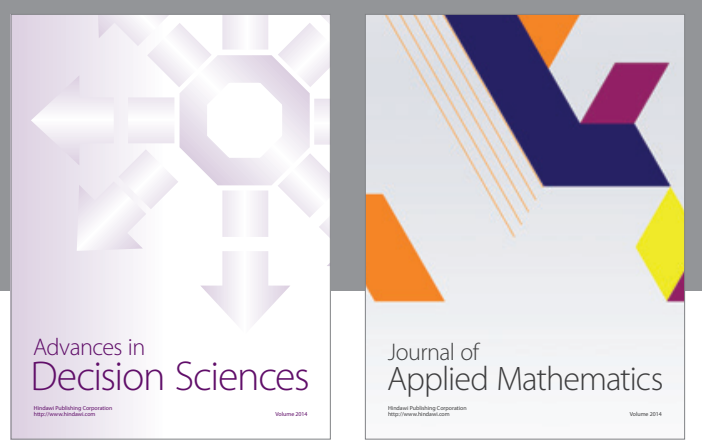

Algebra

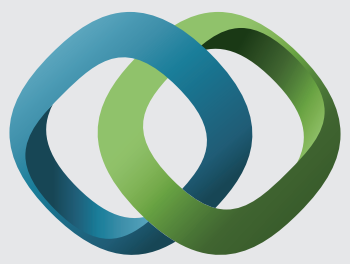

\section{Hindawi}

Submit your manuscripts at

https://www.hindawi.com


Mathematical Problems in Engineering
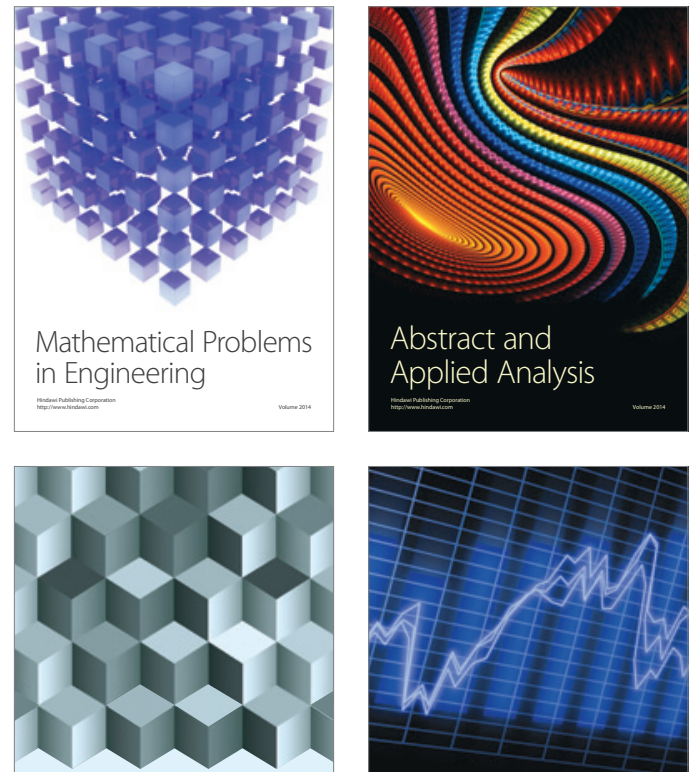

Journal of

Function Spaces

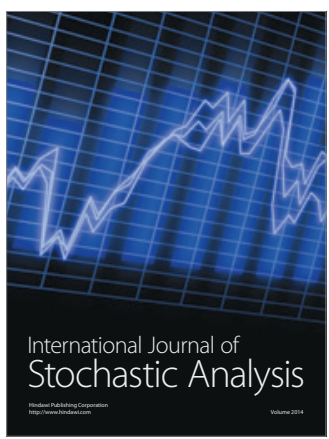

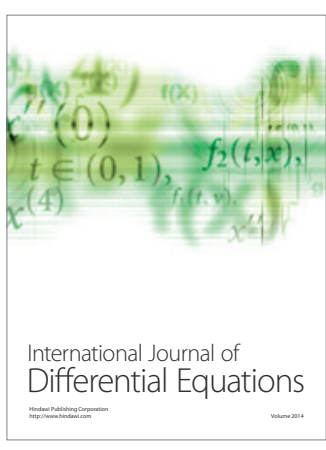
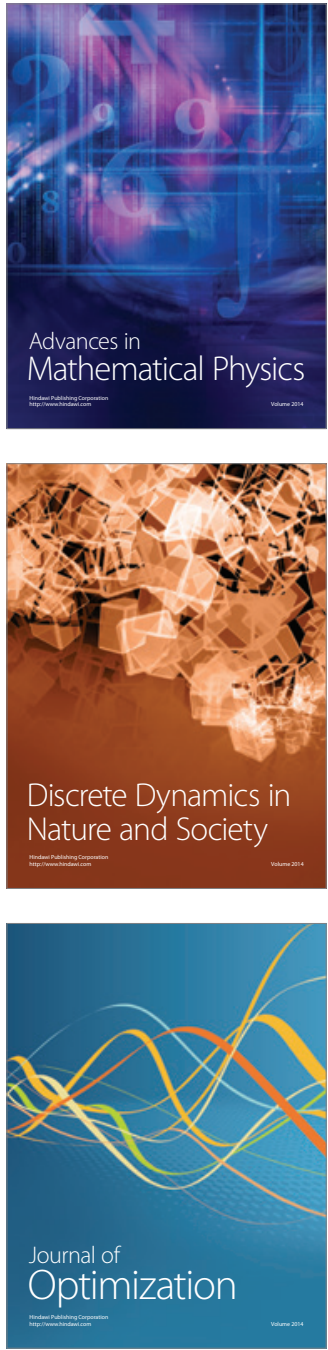